American Journal of Pharmacology and Toxicology 2 (2): 51-59, 2007

ISSN 1557-619X

(C) 2007 Science Publications

\title{
Naloxone benzoylhydrazone activates Extracellular Signal-Regulated Protein Kinases and modulates Nociceptin Opioid Peptide Receptor activity
}

\author{
${ }^{1,2}$ Deepak R. Thakker, ${ }^{1}$ Ghazi F. Altememi, ${ }^{1,3}$ Chitra D. Mandyam, ${ }^{1}$ Hala Ibrahim, \\ ${ }^{1,4}$ Vanessa I. Ramirez and ${ }^{1,4}$ Kelly M. Standifer \\ ${ }^{1}$ Department of Pharmacological and Pharmaceutical Sciences, University of Houston, \\ Houston, TX, 77204-5037 \\ ${ }^{2}$ Biosciences R\&D, Medtronic, Inc., Minneapolis, MN 55432. \\ ${ }^{3}$ Committee on the Neurobiology of Addictive Disorders, The Scripps Research Institute, \\ La Jolla, CA 92037. \\ ${ }^{4}$ University of Oklahoma Health Sciences Center, 1110 N, Stonewall Ave, Suite 329, \\ Oklahoma City, OK 73117
}

\begin{abstract}
Naloxone benzoylhydrazone (NalBzoH) is often used as a nociceptin opioid peptide (NOP) receptor antagonist. However, NalBzoH is also a mixed $\mu$ antagonist $/ \kappa$ and $\delta$ opioid agonist and its actions at the NOP receptor range from an antagonist to partial agonist, depending on the effector response/system studied. We now report that NalBzoH activates endogenously expressed $\kappa_{3}$ opioid, but not NOP, receptors to stimulate extracellular signal-regulated protein kinases $1 / 2$ and, subsequently, to mediate changes in the expression of tyrosine hydroxylase and G protein-coupled receptor kinase 2 in SH-SY5Y human neuroblastoma cells. Furthermore, pretreatment with NalBzoH produces homologous desensitization of the $\kappa_{3}$ opioid receptor as well as heterologous desensitization of the NOP receptor. In contrast, pretreatment with the NOP agonist, orphanin FQ/nociceptin $(\mathrm{OFQ} / \mathrm{N})$, desensitizes only NOP but not the NalBzoH response, suggesting the involvement of a receptor, distinct from NOP, in mediating the actions of NalBzoH in SH-SY5Y cells.
\end{abstract}

Keywords: orphanin $\mathrm{FQ}$ /nociceptin, tyrosine hydroxylase, $\mathrm{G}$ protein-coupled receptor kinase 2

\section{INTRODUCTION}

The multiplicity of opioid receptors and their widespread distribution throughout the central and peripheral nervous systems enables opiates and endogenous opioid peptides to elicit a broad spectrum of pharmacological and physiological actions. While cloning studies provide a general classification of opioid receptors into $\mu, \delta, \kappa$ and nociceptin opioid peptide (NOP) receptors, pharmacological evidence suggests the existence of subtypes for these cloned receptors ${ }^{[1,2]}$. The $\kappa$ opioid receptor has been classified into three subtypes: $\kappa_{1}, \kappa_{2}$ and $\kappa_{3}$. Studies elucidating the actions of $\mathrm{NalBzoH}$ at classical opioid receptor subtypes described it as a mixed $\kappa$ agonist $/ \mu$ antagonist $^{[3-7]}$, as a $\delta$ opioid receptor antagonist with partial agonist actions at the $\kappa_{1}$ opioid receptor ${ }^{[5]}$, and as an agonist at recombinant and olfactory $\delta$ opioid receptors $^{[8,9]}$. Besides demonstrating a potent antagonistic action of $\mathrm{NalBzoH}$ at $\mu$ opioid receptors, the initial in vivo studies revealed that $\mathrm{NalBzoH}$ was an agonist at a novel $\kappa$ opioid receptor and produced analgesia when administered into the cerebral ventricles. Like other opioid agonists, $\mathrm{NalBzoH}$ also inhibited forskolin-stimulated cAMP accumulation, and this was not reversed by selective $\mu-, \delta$ - or $\kappa_{1}$-receptor antagonists ${ }^{[10,11]}$ or by antisense DNA targeting the NOP receptor ${ }^{[12]}$. It was, however, reversed by a less selective $\mu / \kappa$ antagonist, $\operatorname{Mr} 2266^{[10,12]}$. Likewise, NalBzoH-mediated analgesia was not reversed by selective $\mu-, \delta$ - or $\kappa_{1}$-receptor antagonists, and failed to exhibit any cross-tolerance with $\mu$ - or $\kappa_{1}$-agonists ${ }^{[3,4]}$. These data collectively suggested that $\mathrm{NalBzoH}$ activates a novel $\kappa$-receptor subtype, $\kappa_{3}$, which corresponds to the nalorphine $(\mathrm{N})$ receptor proposed by Martin $^{[13]}$.

While the molecular characterization of the $\kappa_{3}$ receptor is not complete, our understanding of the pharmacological actions of NalBzoH has increased. A study performed with NOP receptor-knockout mice suggests that $\mathrm{NalBzoH}$ elicits its analgesic response by antagonizing the hyperalgesic action of the endogenous peptide, $\mathrm{OFQ} / \mathrm{N}$, at the NOP receptor and it was proposed that the $\kappa_{3}$ receptor was the NOP receptor itself $^{[14,15]}$. Several other studies supported the role of $\mathrm{NalBzoH}$ as a competitive antagonist at the NOP receptor ${ }^{[14,16,17]}$, albeit with less potency than its antagonist actions at the $\mu$ receptor ${ }^{[6,18-22]}$. However, other studies strongly negated this relationship. In both rats and mice, an approach using antisense DNA clearly demonstrated that the $\kappa_{3}$ receptor is not identical to NOP, but rather shares a common sequence with it ${ }^{[23-27]}$. Recently, we illustrated differences between NOP and $\kappa_{3}$ opioid receptors in BE(2)-C human neuroblastoma cells $^{12]}$.

Corresponding Author: Kelly M. Standifer, Ph.D., University of Oklahoma Health Sciences Center, Dept. Pharmaceutical Sciences, 1110 N. Stonewall Ave, Suite 329, Oklahoma City, OK 73117.(Phone) 405-271-6593, x47333, (Fax) 405-271-7505 
To further distinguish the actions of $\mathrm{NalBzoH}$ from those of OFQ/N at the cellular level, we examined the activation of extracellular signal-regulated protein kinases 1 and 2 (ERK1/2) by $\kappa_{3}$ and NOP receptors endogenously expressed ${ }^{[11,28-30]}$ in SH-SY5Y human neuroblastoma cells and their resulting effects on levels of tyrosine hydroxylase (TH) and $\mathrm{G}$ protein-coupled receptor kinase (GRK) 2.

\section{MATERIALS AND METHODS}

Materials: The following drugs and materials were purchased from, or kindly provided by, the sources indicated: cell culture media (GibcoBRL, Grand Island, $\mathrm{NY}$ ); fetal bovine sera, penicillin $\mathrm{G}$ and streptomycin sulfate (Atlanta Biologicals, Norcross, GA); NalBzoH and Mr2266 (Dr. Gavril W. Pasternak, Memorial SloanKettering Cancer Center, New York, NY); OFQ/N, Naltriben, DPDPE and CTAP (Research Technology Branch of National Institute on Drug Abuse, Rockville, MD); peptide III BTD (Multiple Peptide Systems, San Diego, CA); PTX (List Biological Laboratories, Campbell, CA); chelerythrine chloride and wortmannin (Research Biochemicals, Inc., Natick, MA); PD98059, phospho-specific ERK1/2, and ERK1/2 antisera (Cell Signaling Technology, Inc., Beverly, MA); $\left[{ }^{3} \mathrm{H}\right] \mathrm{cAMP}$ (Amersham Life Sciences, Arlington Heights, IL); TH and GAPDH antibodies (Chemicon International, Inc., Temecula, CA); GRK2 antisera (sc-562; Santa Cruz Biotechnology, Inc., Santa Cruz, CA). All SDS-PAGE reagents were obtained from Bio-Rad (Hercules, CA) and other chemicals/reagents were purchased from Sigma-Aldrich (St. Louis, MO).

Cell culture and experimental procedures: $\mathrm{SH}-\mathrm{SY} 5 \mathrm{Y}$ neuroblastoma cells (passages 38-52) were cultured and maintained as described ${ }^{[119}$. Cells were grown to 60 $80 \%$ confluence in 24 -well or $100 \mathrm{~cm}^{2}$ dishes in a $6 \%$ $\mathrm{CO}_{2}-94 \%$ air humidified atmosphere at $37^{\circ} \mathrm{C}$. ERK $1 / 2$ activation following short-term agonist exposure or changes in TH and GRK2 proteins after prolonged agonist exposure were measured as described ${ }^{[30,31]}$. The ability of $\mathrm{NalBzoH}$ or $\mathrm{OFQ} / \mathrm{N}$ to inhibit forskolinstimulated cAMP accumulation was measured following drug pretreatment ${ }^{[10]}$, wherein cells were washed in serum-free media and pretreated for $1 \mathrm{~h}$ at $37^{\circ} \mathrm{C}$ with the indicated drug in the same media containing protease-free bovine serum albumin $(0.1 \%)$ and bacitracin $(0.25 \mathrm{mg} / \mathrm{ml})$. NalBzoH was dissolved in $25 \mathrm{mM}$ Tris Citrate, $\mathrm{pH} 5$.

Image and statistical analysis: Following SDSPAGE, immunoreactive bands for ERK, TH, GRK2 and GAPDH were densitized as described ${ }^{[30,31]}$. Phospho/total ERK, TH/GAPDH and GRK2/GAPDH immunoreactivity (IR) ratios were calculated for each band (for statistical analysis) and normalized with respect to basal values (for data presentation). Representative immunoblots were scanned (Hewlett
Packard Scanjet 6300C, with 1200 dpi optical resolution); the resulting images were cropped and sized for figures using Adobe Photoshop, version 5.0 for PC. Concentration-response curves were fitted and $\log \mathrm{EC}_{50}$ values were determined using non-linear regression analysis (GraphPad Prism version 4.00 for Windows; GraphPad Software, San Diego, CA). Statistical comparisons of data were performed with unpaired $t$ test or one-way ANOVA followed by Dunnett's or Tukey's post-hoc test, where appropriate, using GraphPad Prism. Data are expressed as mean \pm s.e.m. unless otherwise indicated and differences were considered significant if $\mathrm{p} \leq 0.05$.

\section{RESULTS}

Time- and concentration-dependent activation of ERK1/2 by NalBzoH: SH-SY5Y cells were stimulated with NalBzoH $(1 \mu \mathrm{M})$ for various time periods ranging from 1-60 min and cell lysates were subjected to SDSPAGE as described in Methods to determine ERK1/2 activation. Maximal ERK1/2 activation (3.1 fold) was achieved in $5 \mathrm{~min}$ (Fig. 1A); phospho/total ERK immunoreactivity (IR) ratios for ERK1 and ERK2 were $3.97 \pm 0.29$ and $3.06 \pm 0.23$ with $\mathrm{NalBzoH}$ versus basal ratios of $1.29 \pm 0.3$ and $0.99 \pm 0.23$, respectively.
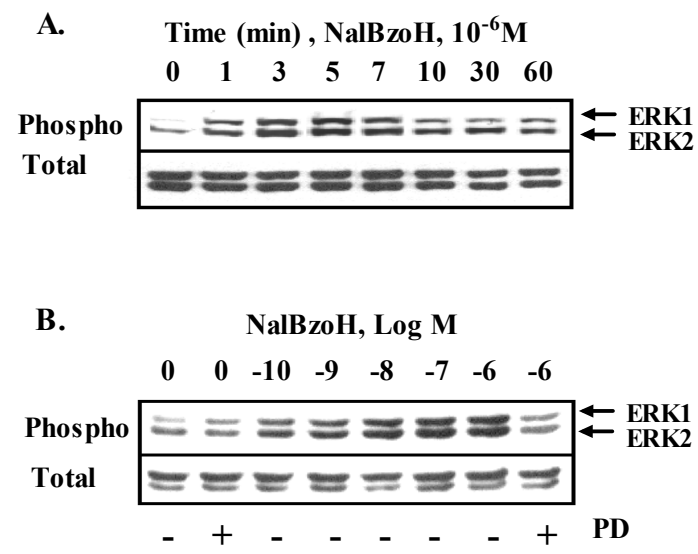

Fig. 1: Time- and concentration-dependent stimulation of ERK1/2 by NalBzoH: Cells were serum-deprived for $24 \mathrm{~h}$ and (A) incubated with $1 \mu \mathrm{M} \mathrm{NalBzoH}$ for the indicated time or (B) were pretreated with or without PD98059 (PD; $10 \mu \mathrm{M})$ for 60 min followed by a $5 \mathrm{~min}$ stimulation with increasing concentrations of NalBzoH as described in Methods. Cell lysates were subjected to SDS-PAGE and membranes were sequentially blotted with phospho-specific ERK1/2 and ERK1/2 antisera. Immunoblots are representative of four to six independent experiments.

Significant ERK1/2 activation persisted for $10 \mathrm{~min}$; thereafter gradually decreasing to 1.4 fold of basal 
levels by 60 min of NalBzoH treatment. Based on this time course study, all subsequent experiments of the acute ERK1/2 activation by NalBzoH were for $5 \mathrm{~min}$. The concentration-response curves for ERK1/2 activation by $\mathrm{NalBzoH}$ reveal an appreciable increase in ERK1/2 activity with drug concentrations as low as 1 $\mathrm{nM}$ and maximal activation by $1 \mu \mathrm{M}$ (Fig. 1B). The $\log \mathrm{EC}_{50}$ values for ERK1 and ERK2 activation are $7.96 \pm 0.16$ and $-8.19 \pm 0.22$, respectively, indicating that $\mathrm{NalBzoH}$ activates both isoforms with similar potency. NalBzoH stimulation of ERK1/2 was completely abolished upon pretreatment with PD 98059 (10 $\mu \mathrm{M}$; Fig. 1B), an inhibitor of mitogen-activated protein kinase kinases 1 and 2 (MEK1/2); immediate upstream activators of ERK1/2. No significant change in total ERK levels was observed with any treatment.

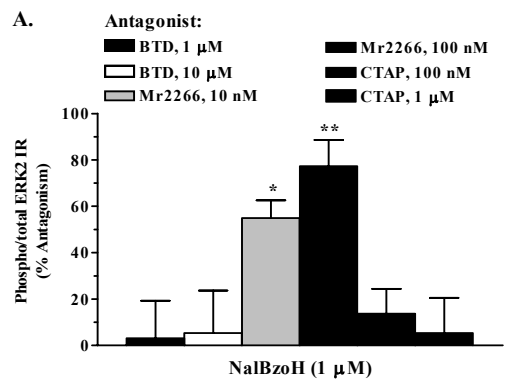

B.

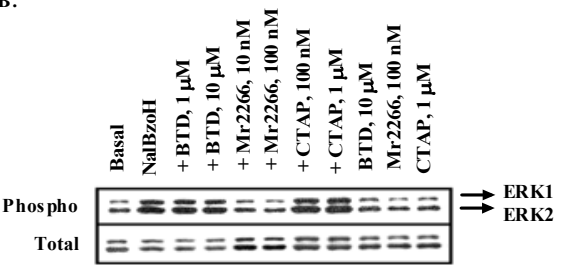

Fig. 2: Antagonism of NalBzoH-mediated ERK1/2 activation. Cells were serum-deprived for $24 \mathrm{~h}$, washed and treated for $5 \mathrm{~min}$ with the indicated concentrations of peptide III BTD (BTD), Mr2266 or CTAP before ERK1/2 stimulation with $1 \mu \mathrm{M}$ $\mathrm{NalBzoH}$ for $5 \mathrm{~min}$. Cell lysates were subjected to immunoblot analysis using phospho-specific ERK1/2 and ERK1/2 antisera as described in Methods. A. Data are compiled by densitometric analysis and represent the mean percentage antagonism of NalBzoH-mediated ERK2 activation $(2.80 \pm 0.09$ fold $) \pm$ s.e.m. values from four independent experiments. $* \mathrm{p}<0.05, * * \mathrm{p}<0.01$; significantly different from $\mathrm{NalBzoH}$-mediated ERK2 activation. B. A representative immunoblot.

NalBzoH stimulates a receptor distinct from NOP or $\mu$ opioid receptors to activate ERK1/2: Among the receptors with which $\mathrm{NalBzoH}$ has been found to interact, $\mu, \delta, \kappa_{3}$ and NOP are present in SH-SY5Y cells $[11,29,32,33]$. Therefore, to determine the receptor/s involved in NalBzoH-mediated ERK1/2 stimulation in this cell line, cells were pretreated with or without peptide III BTD (NOP receptor antagonist ${ }^{[34]}$ ), CTAP $\left(\mu\right.$ receptor antagonist ${ }^{[11]}$ ) or Mr2266 (nonselective, $\mu$ and $\kappa$ receptor antagonist ${ }^{[10,35]}$ ) prior to $\mathrm{NalBzoH}$ challenge. NalBzoH-stimulated ERK1/2 phosphorylation was not antagonized by peptide III BTD or CTAP (Fig. 2). Mr2266, previously shown to block NalBzoHmediated inhibition of cAMP accumulation ${ }^{[10,12]}$, significantly blocked the ERK $1 / 2$ activation induced by NalBzoH in the present study as well (Fig. 2). The antagonists alone did not alter the basal ERK1/2 phosphorylation levels at the concentrations used (Fig. 2B).

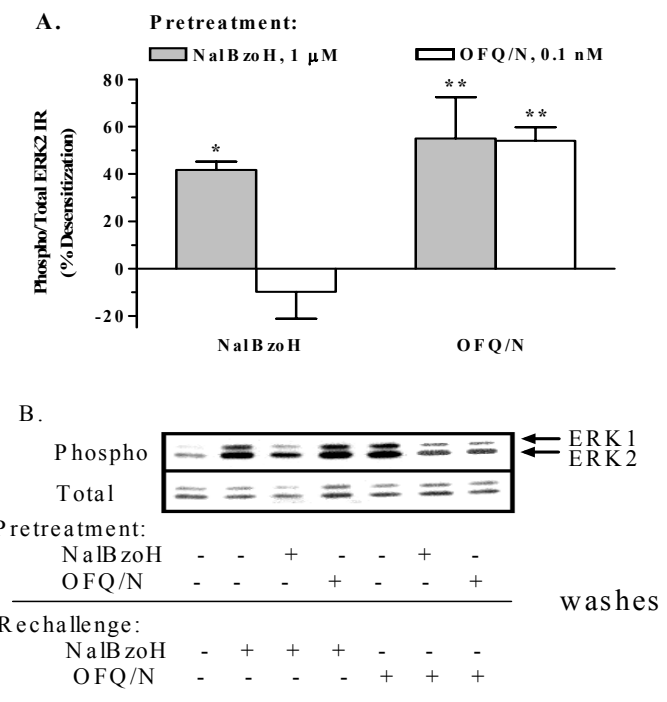

Fig. 3: Effect of agonist pretreatment on subsequent ERK1/2 stimulation by $\mathrm{NalBzoH}$ or OFQ/N. SH-SY5Y cells were serum-deprived for $24 \mathrm{~h}$, washed free of media and pretreated for 5 min with $1 \mu \mathrm{M} \mathrm{NalBzoH}$ ( $\kappa_{3}$ agonist) or $0.1 \mathrm{nM} \mathrm{OFQ} / \mathrm{N}$ (ORL1 agonist). Cells were washed with ice-cold HBSS and re-challenged with NalBzoH $(1 \mu \mathrm{M})$ or OFQ/N $(1 \mathrm{pM})$ for 5 $\min$ at $37^{\circ} \mathrm{C}$. Cell lysates were subjected to SDS-PAGE and membranes were immunoblotted with phospho-specific ERK1/2 and ERK1/2 antisera. A. Data are compiled by densitometric analysis and are presented as mean percentage inhibition of agonist-induced ERK2 activation \pm s.e.m. values from three to four separate experiments. ${ }^{*} \mathrm{p}<0.05$, ${ }^{*} \mathrm{p}<0.01$; significantly different from agonist-mediated ERK2 activation. B. A representative immunoblot.

A receptor desensitization approach was utilized to further explore the site of action of $\mathrm{NalBzoH}$. Cells were pretreated with maximally effective concentrations of $\mathrm{NalBzoH}\left(1 \mu \mathrm{M}^{[10,12]}\right)$ or OFQ/N $(0.1$ $\left.\mathrm{nM}^{[29-31]}\right)$ for 5 min, washed and rechallenged to determine ERK1/2 activation. Stimulating cells with the NOP receptor agonist, OFQ/N (1 pM), for $5 \mathrm{~min}$ induced a $2.66 \pm 0.09$ fold activation of ERK2 as reported earlier ${ }^{[30]}$; NalBzoH induced a $2.71 \pm 0.11$ fold activation of ERK2. Pretreatment with NalBzoH or 
OFQ/N significantly inhibited the subsequent stimulation of ERK $1 / 2$ upon rechallenge with the same drug (Fig. 3), demonstrating that each drug was able to induce homologous desensitization of its own ERK1/2 response. Pretreating the cells with NalBzoH also produced a significant desensitization of $\mathrm{OFQ} / \mathrm{N}$ mediated ERK1/2 activation (Fig. 3). However, pretreatment with $\mathrm{OFQ} / \mathrm{N}$ had no effect on NalBzoHmediated ERK1/2 activation (Fig. 3). This further suggests that $\mathrm{NalBzoH}$ acts at a site distinct from that stimulated by $\mathrm{OFQ} / \mathrm{N}$.

The inhibitory actions of $\mathrm{NalBzoH}$ and $\mathrm{OFQ} / \mathrm{N}$ on adenylyl cyclase also were examined to confirm the interactions suggested by the ERK1/2 activation experiments. Cells were stimulated with NalBzoH (1 $\mu \mathrm{M})$ or $\mathrm{OFQ} / \mathrm{N}(0.1 \mathrm{nM})$ for $1 \mathrm{~h}$, washed extensively, and rechallenged for $10 \mathrm{~min}$ with increasing concentrations of $\mathrm{NalBzoH}$ or $\mathrm{OFQ} / \mathrm{N}$ to test their ability to inhibit forskolin $(10 \mu \mathrm{M})$-stimulated cAMP accumulation. Similar to our previous observation, NalBzoH pretreatment desensitized both OFQ/N- and NalBzoH-mediated inhibition of cAMP accumulation, reducing the maximal response to each by $>50 \%$ (Fig. 4). However, pretreatment with $\mathrm{OFQ} / \mathrm{N}$ desensitized only subsequent OFQ/N-mediated inhibition of cAMP accumulation (by 58\%; Fig. 4). Interestingly, OFQ/N pretreatment significantly increased the potency of NalBzoH to inhibit cAMP accumulation in SH-SY5Y cells as indicated by $\mathrm{EC}_{50}$ shift from $30 \mathrm{nM}$ to $3 \mathrm{nM}$ $(\mathrm{p}<0.0001$; Fig. 4).

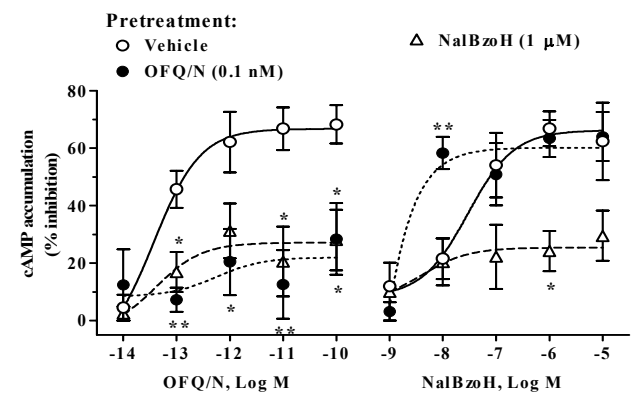

Fig. 4: Effect of agonist pretreatment on subsequent $\mathrm{OFQ} / \mathrm{N}$ - or NalBzoH-mediated inhibition of cAMP accumulation. Cells were incubated for $1 \mathrm{~h}$ with vehicle or with indicated concentrations of $\mathrm{OFQ} / \mathrm{N}$ or $\mathrm{NalBzoH}$ as described in Methods. Intact cells were then assayed for $10 \mathrm{~min}$ with increasing concentrations of $\mathrm{OFQ} / \mathrm{N}$ or $\mathrm{NalBzoH}$ to inhibit forskolin $(10 \mu \mathrm{M})$-stimulated cAMP accumulation. Data represent mean \pm s.e.m. values from four to five independent experiments performed in duplicate. The $\log \mathrm{EC}_{50}$ values for inhibiting forskolin-stimulated cAMP accumulation were $13.43 \pm 0.09(\mathrm{OFQ} / \mathrm{N})$ and $-7.53 \pm 0.15(\mathrm{NalBzoH})$ in vehicle-treated cells. $\quad * \mathrm{p}<0.05, \quad * * \mathrm{p}<0.01 ;$ significantly different from agonist-mediated inhibition of forskolinstimulated cAMP accumulation in vehicle-treated cells.

NalBzoH activates a distinct signaling cascade as compared to $\mathrm{OFQ} / \mathrm{N}$ for stimulating ERK1/2: To examine the signaling components involved in NalBzoH- and OFQ/N-mediated ERK1/2 activation, cells were pretreated with $100 \mathrm{ng} / \mathrm{ml}$ pertussis toxin (PTX) for $24 \mathrm{~h}, 1 \mu \mathrm{M}$ chelerythrine chloride for $5 \mathrm{~min}$ or $100 \mathrm{nM}$ wortmannin for $10 \mathrm{~min}$ prior to agonist challenge. PTX completely attenuated the ERK1/2 signaling by $\mathrm{NalBzoH}$ and $\mathrm{OFQ} / \mathrm{N}$, indicating a role for $\mathrm{G}_{\mathrm{i} / \mathrm{o}}$ proteins in these responses (Fig. 5). The selective protein kinase $\mathrm{C}$ (PKC) inhibitor, chelerythrine, blocked the ERK1/2 activation elicited by NalBzoH and $\mathrm{OFQ} / \mathrm{N}$ by $54 \%$, while the selective phosphatidylinositol 3-kinase (PI3K) inhibitor, wortmannin, blocked only the $\mathrm{OFQ} / \mathrm{N}$ response (by $48 \%$; Fig. 5). None of the inhibitors alone had any effect on basal ERK1/2 activation at the concentrations used (Fig. 5B). Therefore, while NalBzoH-mediated ERK1/2 signaling partly involves the activation of $\mathrm{PKC}, \mathrm{OFQ} / \mathrm{N}$-stimulated signaling is mediated by $\mathrm{PKC}$ and PI3K. These results suggest that the actions of $\mathrm{OFQ} / \mathrm{N}$ involve intracellular signaling cascades distinct from those of NalBzoH.

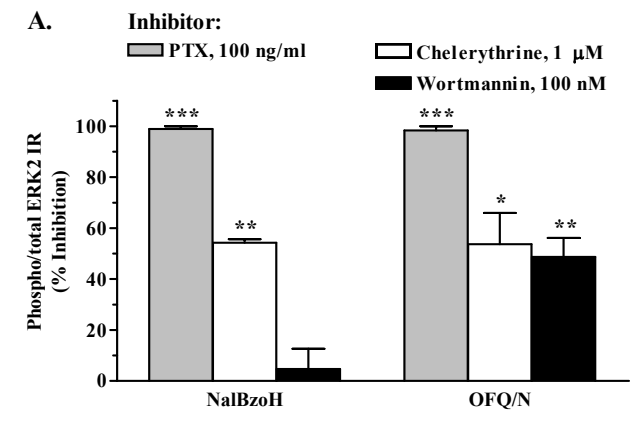

B.

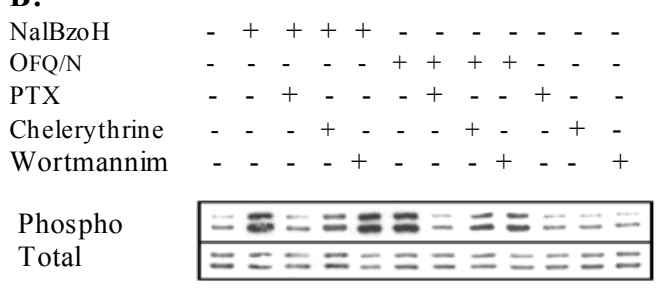

Fig. 5: Blockade of $\kappa_{3}$ or ORL1 receptor-mediated ERK1/2 activation by pertussis toxin and protein kinase inhibitors. SH-SY5Y cells were serum-deprived for $24 \mathrm{~h}$ in the presence or absence of the indicated concentration of pertussis toxin. Cells were washed and pertussis toxin-naive cells were treated with or without the indicated concentrations of chelerythrine (PKC inhibitor) for $5 \mathrm{~min}$ or wortmannin (PI3K inhibitor) for 10 min prior to ERK1/2 stimulation by NalBzoH $(1 \mu \mathrm{M})$ or $\mathrm{OFQ} / \mathrm{N}(1 \mathrm{pM})$. Cell lysates were subjected to SDS-PAGE and membranes were immunoblotted with phospho-specific ERK $1 / 2$ and ERK1/2 antisera. A. Data are compiled by densitometric analysis and are presented as mean percentage inhibition of agonist-induced ERK2 activation (NalBzoH: $3.16 \pm 0.19$ and $\mathrm{OFQ} / \mathrm{N}: 2.95 \pm 0.14$ fold $) \pm$ s.e.m. values from four independent experiments. ${ }^{*} \mathrm{p}<0.05,{ }^{* *} \mathrm{p}<0.01$, ${ }^{* * *} \mathrm{p}<0.001$; significantly different from agonist-mediated ERK2 activation. B. A representative immunoblot. 
Effect of prolonged NalBzoH exposure on endogenous TH and GRK2 levels in SH-SY5Y cells: We have previously demonstrated that prolonged stimulation (24 h) of SH-SY5Y cells with $\mu$ agonists upregulates endogenous $\mathrm{TH}$ levels while stimulation with OFQ/N downregulates TH levels ${ }^{[30]}$. Prolonged treatment with morphine or $\mathrm{OFQ} / \mathrm{N}$ also upregulates endogenous GRK2 levels via activation of ERK1/2 ${ }^{[31]}$. Therefore, we studied the effects of prolonged NalBzoH treatment on TH and GRK2 levels in SHSY5Y cells. Cells were treated with NalBzoH $(1 \mu \mathrm{M})$ for $24 \mathrm{~h}$ and cell lysates were subjected to SDS-PAGE to detect $\mathrm{TH}$ and GRK2 levels. Prolonged NalBzoH exposure significantly increased the levels of TH (Fig. 6) and GRK2 (Fig. 7); this was blocked upon inclusion

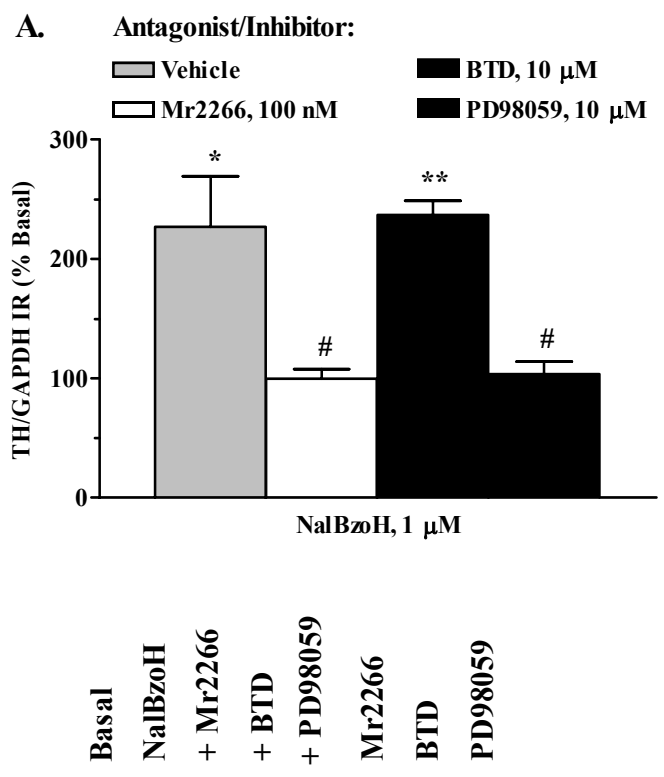

B.

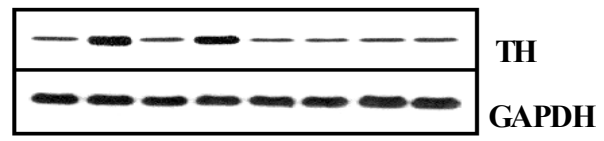

Fig. 6: TH induction following prolonged activation of the $\kappa_{3}$ receptor is mediated via ERK1/2. Cells were pretreated with or without indicated concentrations of Mr2266 or BTD for 5 min or PD98059 for $60 \mathrm{~min}$ prior to stimulation with $1 \mu \mathrm{M}$ $\mathrm{NalBzoH}$ for $24 \mathrm{~h}$ as described in Methods. Cell lysates were immunoblotted with $\mathrm{TH}$ and GAPDH antibodies. A. Densitometric analysis of immunoreactive bands represents the mean percentage increase \pm s.e.m. values over basal TH/GAPDH ratio $(0.86 \pm 0.35)$ from four to six independent experiments. ${ }^{*} \mathrm{p}<0.05,{ }^{* *} \mathrm{p}<0.01$; significantly different from basal and ${ }^{\#}<0.05$; significantly different from NalBzoHinduced TH levels. B. A representative immunoblot. of the $\mu / \kappa$ receptor antagonist, Mr2266, but not the NOP receptor antagonist, peptide III BTD (Figs. 6 and 7). In contrast, OFQ/N-mediated GRK2 upregulation was reversed by peptide III BTD, but not Mr2266 (Fig. 7). As a control to determine if activation of $\delta$ opioid receptors stimulates upregulation of GRK2 and TH in SH-SY5Y cells, cells were treated with the full $\delta$ opioid agonist, DPDPE $(1 \mu \mathrm{M})$ for $24 \mathrm{hr}$. Treatment with DPDPE failed to significantly increase levels of GRK2 $(108.4 \pm 13.6 \%$ Basal; $n=5)$ or $\mathrm{TH}(100.4 \pm 19.6 \%$ Basal; $n=5)$.

Treating the cells with $\mathrm{NalBzoH}$ in the presence of PD98059 $(10 \mu \mathrm{M})$ completely abolished NalBzoH-induced upregulation of both, TH and GRK2 (Figs. 6 and 7), indicating the involvement of ERK $1 / 2$ in both events. Neither PD98059 nor the inhibitors alone had a significant effect on basal TH or GRK2 levels (Figs. 6B and 7B).
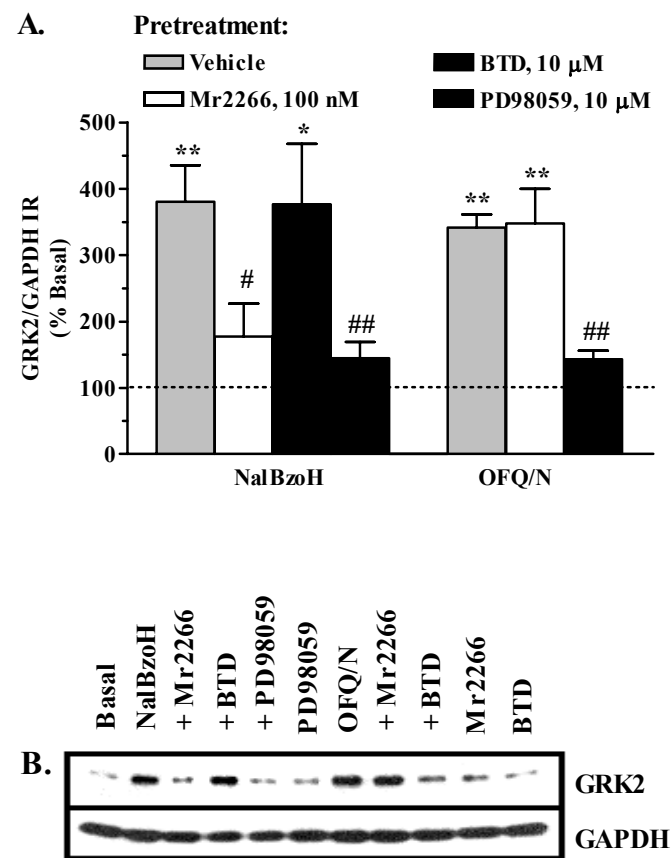

Fig. 7: Prolonged exposure to NalBzoH induces GRK2 levels via a receptor distinct from ORL1 via involvement of ERK1/2. Cells were pretreated with or without indicated concentrations of Mr2266 or BTD for $5 \mathrm{~min}$ or PD98059 for $60 \mathrm{~min}$ prior to stimulation with NalBzoH $(1 \mu \mathrm{M})$ or OFQ/N $(0.1 \mathrm{nM})$ for 24 $\mathrm{h}$ as described in Methods. Cell lysates were immunoblotted with GRK2 and GAPDH antibodies. A. Densitometric analysis of immunoreactive bands is presented as the mean percentage increase \pm s.e.m. values over basal GRK2/GAPDH ratio $(0.47 \pm 0.06)$ from three to four separate experiments. $* \mathrm{p}<0.05, * * \mathrm{p}<0.01$; significantly different from basal and ${ }^{\#} \mathrm{p}<0.05,{ }^{\#} \mathrm{p}<0.01$; significantly different from druginduced GRK2 levels. B. A representative immunoblot. 


\section{DISCUSSION}

In the present study, we provide further evidence suggesting that $\mathrm{NalBzoH}$ activates the $\kappa_{3}$ opioid receptor and that it upregulates TH and GRK2 protein expression via stimulation of ERK1/2 through the $\kappa_{3}$ opioid receptor. Several lines of evidence indicate that $\mathrm{NalBzoH}$ activates a receptor distinct from the NOP receptor to stimulate ERK1/2 activity in SHSY5Y cells. Functionally, NalBzoH antagonizes the actions of $\mu$ opioid receptor agonists ${ }^{[3-5,10]}$ and, therefore is unlikely to activate ERK $1 / 2$ via the $\mu$ opioid receptor in this cell line. In $\mathrm{CHO}$ cells expressing recombinant $\mu$ opioid receptor, $\mathrm{NalBzoH}$ failed to activate ERK1/2 or inhibit cAMP (data not shown). We do not observe any significant ERK1/2 activation in SH-SY5Y cells following exposure to selective $\mu$ (CTAP) or NOP (peptide III BTD) receptor antagonists at concentrations effective for blocking the actions of respective receptor agonists. These antagonists failed to inhibit NalBzoH-stimulated ERK1/2 activation (Fig. 2), suggesting that neither $\mu$ nor NOP receptors were mediating the NalBzoH response in SH-SY5Y cells. Previous studies have clearly ruled out the involvement of the $\delta$ opioid receptor, as its antagonists had no effect on the actions of $\mathrm{NalBzoH}^{[4,10]}$. Mr2266, a $\mu / \kappa$ receptor antagonist ${ }^{[35]}$, was previously found to inhibit $\kappa_{3}$, but not NOP, receptor signaling ${ }^{[10,12]}$. In the present study also, Mr2266 blocked the ERK1/2 phosphorylation resulting from $\mathrm{NalBzoH}$, but not OFQ/N, exposure.

The conclusion that NalBzoH elicits its actions via a distinct receptor also is suggested by our observation that pretreatment with the NOP receptor agonist, $\mathrm{OFQ} / \mathrm{N}$, produced homologous desensitization of its subsequent ERK1/2 response but had no effect on NalBzoH-mediated ERK1/2 activation. Pretreatment with $\mathrm{NalBzoH}$ significantly diminished subsequent NalBzoH-stimulated ERK1/2 activation, confirming a previous report ${ }^{[12]}$ that the $\kappa_{3}$ receptor is subject to homologous desensitization. $\mathrm{NalBzoH}$ pretreatment also attenuated subsequent OFQ/N-mediated ERK1/2 stimulation in SH-SY5Y cells. To determine whether this one-way cross-tolerance was specific to ERK1/2 phosphorylation or could be generalized to other functions, we also examined the inhibition of forskolinstimulated cAMP accumulation. As observed earlier, pretreatment with both $\mathrm{OFQ} / \mathrm{N}$ and $\mathrm{NalBzoH}$ produced a homologous desensitization of their own cAMP inhibitory responses. NalBzoH pretreatment also desensitized NOP-mediated inhibition of forskolinstimulated cAMP accumulation in SH-SY5Y cells. In those systems in which NalBzoH antagonized NOP receptor actions, it did so via competitive binding to the NOP receptor ${ }^{[6,18]}$. This is highly unlikely in our studies as after NalBzoH pretreatment cells were extensively washed before $\mathrm{OFQ} / \mathrm{N}$ application. Given the documented low affinity of NalBzoH for the NOP receptor, continued $\mathrm{NalBzoH}$ association with the receptor is unlikely. An increase in NalBzoH responsiveness following $\mathrm{OFQ} / \mathrm{N}$ pretreatment was previously reported in $\mathrm{BE}(2)-\mathrm{C}$ cells ${ }^{[12]}$. We also observed that $\mathrm{OFQ} / \mathrm{N}$ pretreatment increases the potency of NalBzoH for inhibiting forskolin-stimulated cAMP accumulation in SH-SY5Y cells. Though suggestive, the increase in NalBzoH-mediated ERK1/2 activation following $\mathrm{OFQ} / \mathrm{N}$ pretreatment did not reach statistical significance. The desensitization studies provide substantial evidence for distinguishing the actions of $\mathrm{NalBzoH}$ at a distinct receptor site from that activated by $\mathrm{OFQ} / \mathrm{N}$.

NalBzoH-mediated ERK1/2 activation is sensitive to PTX, suggesting the involvement of $\mathrm{G}_{\mathrm{i} / \mathrm{o}}$ proteins. $\mathrm{G}_{\mathrm{i} / \mathrm{o}}$ proteins are also involved in the analgesic action of $\mathrm{NalBzoH}^{[36]}$ as well as in its inhibition of forskolin-stimulated cAMP accumulation in $\mathrm{BE}(2)-\mathrm{C}$ cells ${ }^{[10]}$. $G_{i}$-mediated ERK signaling utilizes the $\beta \gamma$ subunit of $\mathrm{G}_{\mathrm{i}}$ and is PI3K, but not PKC-dependent, whereas $G_{0}$-mediated ERK activation involves the $\alpha$ subunit of $G_{0}$ along with PKC, but not PI3K ${ }^{[37]}$. NalBzoH-stimulated ERK1/2 activity was partially blocked by the PKC, but not the PI3K, inhibitor indicating the involvement of $\mathrm{G}_{0}$, but not $\mathrm{G}_{\mathrm{i}}$, in this response. $\mathrm{OFQ} / \mathrm{N}$-mediated ERK1/2 signaling involves $\mathrm{G}_{\mathrm{i}}$ and $\mathrm{G}_{\mathrm{o}}$ proteins, and is partly mediated by PI3K as well as PKC as reported earlier ${ }^{[38]}$. It is possible that NalBzoH-mediated desensitization of ORL1 occurs as a result of PKC activation, since the NOP receptor is sensitive to $\mathrm{PKC}^{[39-41]}$.

In the present study, we observe that prolonged treatment with $\mathrm{NalBzoH}$ results in an ERK1/2-dependent induction of TH in SH-SY5Y cells, similar to that previously noted for $\mu$ agonists ${ }^{[30,42,43]}$. This is in direct contrast to prolonged OFQ/N exposure that significantly reduces TH levels in SH-SY5Y cells $^{[30]}$. NalBzoH-mediated upregulation of GRK2 levels in an ERK1/2-dependent fashion is similar to that seen with morphine and OFQ/N in SH-SY5Y cells ${ }^{[31]}$. The distinction, however, is that Mr2266, but not peptide III-BTD, blocked TH as well as GRK2 upregulation induced by $\mathrm{NalBzoH}$, again indicating $\kappa_{3}$, and not NOP receptor-mediated effects. Moreover, treatment of cells for $24 \mathrm{hr}$ with the $\delta$-selective opioid agonist DPDPE $(1 \mu \mathrm{M})$ failed to increase GRK2 and/or TH levels, indicating that NalBzoH's actions could not have been mediated through a $\delta$ opioid receptor, and further support our hypothesis that NalBzoH's actions are mediated through a distinct $\kappa_{3}$ opioid receptor.

In the present study, we illustrate for the first time that $\mathrm{NalBzoH}$ activates ERK1/2 through an opioid 
receptor distinct from $\mu, \delta$, and NOP, and demonstrate the potential significance of this signal in contributing to the chronic actions of NalBzoH. Although NalBzoH is not used clinically, other clinically useful agents such as levorphanol and nalbuphine also exhibit $\kappa_{3}$ agonist properties $^{[44,45]}$ and may produce actions similar to $\mathrm{NalBzoH}$ with prolonged administration. We provide further evidence suggesting that $\mathrm{NalBzoH}$ elicits its agonist actions via a receptor distinct from that activated by $\mathrm{OFQ} / \mathrm{N}$, by demonstrating the differential sensitivity of $\mathrm{OFQ} / \mathrm{N}$ and $\mathrm{NalBzoH}$ to receptor antagonists, kinase inhibitors and agonist pretreatments. However, in accordance with previous reports of NalBzoH interacting with the NOP receptor ${ }^{[6,18]}$ as well as the $\mu$ opioid receptor ${ }^{[3-7]}$, it is possible that NalBzoH activates a dimer resulting from association of the two receptors or their splice variants ${ }^{[46,47]}$. Indeed, $\mathrm{NalBzoH}$ affinity for the NOP receptor was increased in cell membranes containing the $\mu$-NOP receptor dimer ${ }^{[46]}$. Whether these dimers are functionally distinct from either receptor alone and are responsible for the actions of NalBzoH still remains to be elucidated. Nevertheless, the present study underscores the need to be cognizant of the many diverse actions of $\mathrm{NalBzoH}$ and other drugs with $\kappa_{3}$ opioid agonist properties when utilizing these drugs in acute as well as long-term studies. Therefore, further examination of the $\kappa_{3}$ versus NOP receptor properties of these agents and their cellular consequences is highly warranted.

\section{ACKNOWLEDGMENTS}

This work was supported, in part, by grants from the U.S. Public Health Service to C.D.M. (F31DA14171) and K.M.S. (R29DA10738 and R01DA017380), and the Texas Advanced Research Programs (003652-01141999 and 003652-0182-2001) to K.M.S.

\section{REFERENCES}

1. Pasternak, G. W., Standifer, K. M., 1995. Mapping of opioid receptors using antisense oligodeoxynucleotides: correlating their molecular biology and pharmacology. Trends in Pharmacol Sci 16:344-350.

2. Mogil, J. S., Pasternak, G. W., 2001. The molecular and behavioral pharmacology of the orphanin $\mathrm{FQ}$ /nociceptin peptide and receptor family. Pharmacol Rev 53:381-415.

3. Gistrak, M. A., Paul, D., Hahn, E. F., Pasternak, G. W., 1989. Pharmacological actions of a novel mixed opiate agonist/antagonist: naloxone benzoylhydrazone. J Pharmacol Exp Ther 251:469476.
4. Paul, D., Levison, J. A., Howard, D. H., Pick, C. G., Hahn, E. F., Pasternak, G. W., 1990. Naloxone benzoylhydrazone (NalBzoH) analgesia. J Pharmacol Exp Ther 255:769-774.

5. Berzetei-Gurske I.P., White, A., Polgar, W., DeCosta, B.R., Pasternak, G.W., Toll, L., 1995. The in vitro pharmacological characterization of naloxone benzoylhydrazone. Eur J Pharmacol 277:257-63.

6. Chiou, L.-C., 2001. Differential antagonism by naloxone benzoylhydrazone of the activation of inward rectifying $\mathrm{K}+$ channels by nociceptin and a mu-opioid in rat periaqueductal grey slices. Naunyn-Schmiedeberg's Arch Pharmacol 363:583589.

7. Brown, G. P., Pasternak, G. W., 1998. ${ }^{3} \mathrm{H}$-naloxone benzoylhydrazone binding in MOR-1-transfected Chinese hamster ovary cells: evidence for Gprotein-dependent antagonist binding. J Pharmacol Exp Ther 286:376-381.

18. Onali P., Olianas, M.C., 2004. G protein activation and cyclic AMP modulation by naloxone benzoylhydrazone in distinct layers of rat olfactory bulb. Br J Pharmacol 143:638-48.

9. Olianas, M.S., Concas, D., Onali, P., 2006. Agonist activity of naloxone benzoylhydrazone at recombinant and native opioid receptors. $\mathrm{Br} \mathrm{J}$ Pharmacol 147:360-70.

10. Standifer, K. M., Cheng, J., Brooks, A. I., Honrado, C. P., Su, W., Visconti, L. M., Biedler, J. L., Pasternak G. W., 1994. Biochemical and pharmacological characterization of mu, delta and kappa $_{3}$ opioid receptors expressed in BE(2)-C neuroblastoma cells. J Pharmacol Exp Ther 270:1246-1255.

11. Cheng, J., Standifer, K. M., Tublin, P. R., Su, W., Pasternak, G. W., 1995. Demonstration of kappa $3^{-}$ opioid receptors in the SH-SY5Y human neuroblastoma cell line. J Neurochem 65:170-175.

12. Mathis, J. P., Mandyam, C. D., Altememi, G. F., Pasternak, G. W., Standifer, K. M., 2001. Orphanin $\mathrm{FQ}$ /nociceptin and naloxone benzoylhydrazone activate distinct receptors in BE(2)-C neuroblastoma cells. Neurosci Lett 299:173-176.

13. Martin, W. R., 1967. Opioid antagonists. Pharmacol Rev 19:463-521.

14. Noda, Y., Mamiya, T., Nabeshima, T., Nishi, M., Higashioka, M., Takeshima, H., 1998. Loss of antinociception induced by naloxone benzoylhydrazone in nociceptin receptor-knockout mice. J Biol Chem 273:18047-18051.

15. Wollemann, M., 1996. Orphan receptor or kappa3opioid receptor? Trends Pharmacol. Sci. 17:217. 
16. Abdulla F.A., Smith, P.A., 1998. Axotomy reduces the effect of analgesic opioids yet increases the effect of nociceptin on dorsal root ganglion neurons. J Neurosci 18:9685-94.

17. Seki, T., Awamura, S. Kimura, C., Ide, S., Sakano, K., Minami, M, Bagase, H., Satoh, M., 1999. Pharmacological properties of TRK- 820 on cloned $\mu-, \delta$-, $\kappa$-opioid receptors and nociceptin receptor. Eur J Pharmacol 376:159-67.

18. Bigoni, R., Calo, G., Rizzi, A., Okawa, H., Regoli, D., Smart, D., Lambert, D. G., 2002. Effects of naloxone benzoylhydrazone on native and recombinant nociceptin/orphanin FQ receptors. Can J Physiol Pharmacol 80:407-412.

19. Kim, K. W., Chung, Y. J., Han, J. H., Woo, R. S., Park, E. Y., Seul, K. H., Kim, S. Z., Cho, K. W., Kim, S. H., 2002. Nociceptin/orphanin FQ increases ANP secretion in neonatal cardiac myocytes. Life Sci 70:1065-1074.

20. Ichikawa, D., Ozaki, S., Azuma, T., Nambu, H., Kawamoto, H., Iwasawa, Y., Takeshima, H., Ohta, H., 2001. In vitro inhibitory effects of J-113397 on nociceptin/orphanin FQ-stimulated. Neuroreport 12, 1757-1761.

21. Sbrenna, S., Marti, M., Morari, M., Calo, G., Guerrini, R., Beani, L., Bianchi, C., 2000. Modulation of 5-hydroxytryptamine efflux from rat cortical synaptosomes by opioids and nociceptin. Br J Pharmacol 130:425-433.

22. Ozaki, S., Kawamoto, H., Itoh, Y., Miyaji, M., Azuma, T., Ichikawa, D., Nambu, H., Iguchi, T., Iwasawa, Y., Ohta, H., 2000. In vitro and in vivo pharmacological characterization of J-113397, a potent and selective non-peptidyl ORL1 receptor antagonist. Eur J Pharmacol 402:45-53.

23. Kolesnikov, Y. A., Pasternak, G. W., 1999. Peripheral orphanin $\mathrm{FQ}$ /nociceptin analgesia in the mouse. Life Sci 64:2021-2028.

24. Pan, Y.-X., Cheng, J., Xu, J., Rossi, G., Jacobson, E., Ryan-Moro, J., Brooks, A. I., Dean, G. E., Standifer, K. M., Pasternak, G. W., 1995. Cloning and functional characterization through antisense mapping of a $\mathrm{kappa}_{3}$-related opioid receptor. Mol Pharmacol 47:1180-1188.

25. Rossi, G. C., Leventhal, L., Bolan, E., Pasternak, G. W., 1997. Pharmacological characterization of orphanin $\mathrm{FQ} /$ nociceptin and its fragments. J Pharmacol Exp Ther 282:858-865.

26. Rossi, G. C., Perlmutter, M., Leventhal, L., Talatti, A., Pasternak, G. W., 1998. Orphanin $\mathrm{FQ}$ /nociceptin analgesia in the rat. Brain Res 792:327-330.
27. King, M. A., Rossi, G. C., Chang, A. H., Williams, L., Pasternak, G. W., 1997. Spinal analgesic activity of orphanin $\mathrm{FQ} /$ nociceptin and its fragments. Neurosci Lett 223:113-116.

28. Wu, Y. L., Pu, L., Ling, K., Zhao, J., Cheng, Z. J., Ma, L., Pei, G., 1997. Molecular characterization and functional expression of opioid receptor-like 1 receptor. Cell Res 7:69-77.

29. Peluso, J., Gaveriaux-Ruff, C., Matthes, H. W., Filliol, D., Kieffer, B. L., 2001. Orphanin $\mathrm{FQ}$ /nociceptin binds to functionally coupled ORL1 receptors on human immune cell lines and alters peripheral blood mononuclear cell proliferation. Brain Res Bull 54:655-660.

30. Thakker, D. R. Standifer, K. M., 2002. Orphanin $\mathrm{FQ}$ /nociceptin blocks chronic morphine-induced tyrosine hydroxylase upregulation. Mol Brain Res 105:38-46.

31. Thakker, D. R., Standifer, K. M., 2002. Induction of $\mathrm{G}$ protein-coupled receptor kinases 2 and 3 contributes to the cross-talk between mu and ORL1 receptors following prolonged agonist exposure. Neuropharmacol 43:979-990.

32. Kazmi, S. M., Mishra, R. K., 1986. Opioid receptors in human neuroblastoma SH-SY5Y cells: evidence for distinct morphine $(\mathrm{mu})$ and enkephalin (delta) binding sites. Biochem Biophys Res Comm 137:813-820.

33. Connor, M., Yeo, A., Henderson, G., 1996. The effect of nociceptin on $\mathrm{Ca} 2+$ channel current and intracellular $\mathrm{Ca} 2+$ in the SH-SY5Y human neuroblastoma cell line. Br J Pharmacol 118:205207.

34. Becker, J.A.J., Wallace, A., Garzon, A., Ingallinella, P., Bianchi, E., Cortese, R., Simonin, F., Kieffer, B.L., Pessi, A., 1999. Ligands for kappa-opioid and ORL1 receptors identified from a conformationally constrained peptide combinatorial library. J Biol Chem 274:2751327522.

35. Calcagnetti, D.J., Calcagnetti, R.L., Fanselow, M.S., 1990. Centrally administered opioid antagonists, nor-binaltorphimine, 16-methyl cyprenorphine and MR2266, suppress intake of a sweet solution. Pharmacol. Biochem. Behav. 35:69-73.

36. Standifer, K. M., Rossi, G. C., Pasternak, G. W., 1996. Differential blockade of opioid analgesia oligodeoxynucleotides directed against various $\mathrm{G}$ protein alpha subunits. Mol. Pharmacol. 50:293298. 
37. Hawes, B. E., Graziano, M. P., Lambert, D. G., 2000. Cellular actions of nociceptin: transduction mechanisms. Peptides 21:961-967.

38. Fukuda, K., Shoda, T., Morikawa, H., Kato, S., Mori, K., 1997. Activation of mitogen-activated protein kinase by the nociceptin receptor expressed in Chinese hamster ovary cells. FEBS Lett 412:290-294.

39. Mandyam, C. D., Thakker, D. R., Christensen, J. L., Standifer, K. M., 2002. Orphanin FQ/Nociceptinmediated desensitization of opioid receptor-like 1 receptor and mu opioid receptors involves protein kinase $\mathrm{C}$ : a molecular mechanism for heterologous cross-talk. J Pharmacol Exp Ther 302:502-509.

40. Pu L., Bao, G. B., Ma, L., Pei, G., 1999. Acute desensitization of nociceptin/orphanin FQ inhibition of voltage-gated calcium channels in freshly dissociated hippocampal neurons. Eur J Neurosci1 1:3610-3616.

41. Pei, G., Ling, K., Pu, L., Cunningham, M. D., Ma, L., 1997. Nociceptin/orphanin FQ stimulates extracellular acidification and desensitization of the response involves protein kinase C. FEBS Lett 412:253-256.
42. Berhow, M.T., Hiroi, N., Nester, E.J., 1996. Regulation of ERK (extracellular signal-regulated kinase), part of the neurotrophin signal transduction cascade, in the rat mesolimbic dopamine system by chronic exposure to morphine or cocaine. J Neurosci16:4707-4715.

43. Boundy, V. A., Gold, S. J., Messer, C. J., Chen, J., Son, J. H., Joh, T. H., Nestler, E. J., 1998. Regulation of tyrosine hydroxylase promoter activity by chronic morphine in TH9.0-LacZ transgenic mice. J Neurosci 18:989-9995.

44. Pick, C. G., Paul, D., Pasternak, G. W., 1992. Nalbuphine, a mixed kappa 1 and 3 analgesia in mice, J Pharmacol Exp Ther 262:1044-1050.

45. Tive, L., Ginsberg, K., Pick, C. G., Pasternak, G. W., 1992. Kappa3 receptors and levorphanolinduced analgesia. Neuropharmacol 31:851-856.

46. Pan, Y.-X., Bolan, E. A., Pasternak, G. W., 2002. Dimerization of morphine and orphanin FQ/nociceptin receptors: generation of a novel opioid receptor subtype. Biochem Biophys Res Comm 297:659-663.

47. Wang, H.L., Hsu, c.Y., Huang, P.C., Huo, Y.L., Li, A.H., Yeh, T.H., Tso, A.S., Chen, Y.L. (2005) Heterodimerization of opioid receptor-like1 and $\mathrm{mu}$ opioid receptors impairs the potency of $\mu$ opioid receptor agonists. J Neurochem 92:1285-94. 